\title{
HIV infection drives interferon signaling within intestinal SARS-CoV-2 target cells
}

\author{
Rabiah Fardoos, ${ }^{1,2}$ Osaretin E. Asowata, ${ }^{1,3}$ Nicholas Herbert, ${ }^{1,3}$ Sarah K. Nyquist, ${ }^{4,5,6,7}$ Yenzekile \\ Zungu, ${ }^{1,3}$ Alveera Singh, ${ }^{1}$ Abigail Ngoepe, ${ }^{1}$ Ian M. Mbano, ${ }^{1,3}$ Ntombifuthi Mthabela, ${ }^{1}$ Dirhona Ramjit, ${ }^{1}$ \\ Farina Karim, ${ }^{1}$ Warren Kuhn, ${ }^{8}$ Fusi G. Madela, ${ }^{9}$ Vukani T. Manzini, ${ }^{9}$ Frank Anderson, ${ }^{9}$ Bonnie Berger, ${ }^{10}$ \\ Tune H. Pers, ${ }^{11}$ Alex K. Shalek, ${ }^{4,5,6}$ Alasdair Leslie, ${ }^{1,3,12}$ and Henrik N. Kløverpris ${ }^{1,2,3,12}$ \\ ${ }^{1}$ Africa Health Research Institute, Durban, South Africa. ${ }^{2}$ Department of Immunology and Microbiology, University of \\ Copenhagen, Copenhagen, Denmark. ${ }^{3}$ School of Laboratory Medicine and Medical Sciences, University of KwaZulu-Natal, \\ Durban, South Africa. ${ }^{4}$ Institute for Medical Engineering \& Science, Department of Chemistry, and Koch Institute for \\ Integrative Cancer Research, Massachusetts Institute of Technology (MIT), Cambridge, Massachusetts, USA. ${ }^{5}$ Broad \\ Institute of MIT and Harvard, Cambridge, Massachusetts, USA. ${ }^{6}$ Ragon Institute of MGH, MIT and Harvard, Cambridge, \\ Massachusetts, USA. ${ }^{7}$ Program in Computational and Systems Biology, MIT, Cambridge, Massachusetts, USA. ${ }^{8}$ ENT \\ Department, General Justice Gizenga Mpanza Regional Hospital (Stanger Hospital), University of KwaZulu-Natal, Durban, \\ South Africa. ${ }^{9}$ Discipline of General Surgery, Inkosi Albert Luthuli Central Hospital, University of KwaZulu-Natal, Durban, \\ South Africa. ${ }^{10}$ Computer Science and Artificial Intelligence Laboratory and Department of Mathematics, MIT, Cambridge, \\ Massachusetts, USA. "Novo Nordisk Foundation Center for Basic Metabolic Research, Faculty of Health and Medical \\ Sciences, University of Copenhagen, Copenhagen, Denmark. ${ }^{12}$ Division of Infection and Immunity, University College \\ London, London, United Kingdom.
}

SARS-CoV-2 infects epithelial cells of the human gastrointestinal (CI) tract and causes related symptoms. HIV infection impairs gut homeostasis and is associated with an increased risk of COVID-19 fatality. To investigate the potential link between these observations, we analyzed singlecell transcriptional profiles and SARS-CoV-2 entry receptor expression across lymphoid and mucosal human tissue from chronically HIV-infected individuals and uninfected controls. Absorptive gut enterocytes displayed the highest coexpression of SARS-CoV-2 receptors ACE2, TMPRSS2, and TMPRSS4, of which ACE2 expression was associated with canonical interferon response and antiviral genes. Chronic treated HIV infection was associated with a clear antiviral response in gut enterocytes and, unexpectedly, with a substantial reduction of ACE2 and TMPRSS2 target cells. Gut tissue from SARS-CoV-2-infected individuals, however, showed abundant SARS-CoV-2 nucleocapsid protein in both the large and small intestine, including an HIV-coinfected individual. Thus, upregulation of antiviral response genes and downregulation of ACE2 and TMPRSS2 in the GI tract of HIV-infected individuals does not prevent SARS-CoV-2 infection in this compartment. The impact of these HIVassociated intestinal mucosal changes on SARS-CoV-2 infection dynamics, disease severity, and vaccine responses remains unclear and requires further investigation.

Authorship note: RF and OEA contributed equally to this work.

Conflict of interest: The authors have declared that no conflict of interests exists.

Copyright: ( 2021 , Fardoos et al. This is an open access article published under the terms of the Creative Commons Attribution 4.0 International License.

Submitted: May 27, 2021

Accepted: July 8, 2021

Published: August 23, 2021

Reference information: /CI Insight. 2021;6(16):e148920. https://doi.org/10.1172/jci. insight.148920.

\section{Introduction}

Gastrointestinal (GI) tract symptoms are observed in up to $60 \%$ of COVID-19 patients and precede respiratory symptoms (1). SARS-CoV-2 can be detected within intestinal tissues $(2,3)$, and about $30 \%$ of COVID-19 patients harbor detectable viral RNA in their stool (4), which is associated with more severe GI symptoms (1). The observed GI disorders include vomiting, nausea, and diarrhea; can manifest local and systemic disease; and may lead to fecal-oral transmission of virus $(2,5,6)$. High expression of ACE2, the primary receptor for SARS-CoV-2, is found on the luminal surface of differentiated small intestinal epithelial cells, whereas crypt-based cells express lower levels $(7,8)$. Studies of human small intestinal organoids show that the mature enterocytes are the major source for SARS-CoV-2 replication $(2,6,9)$. These cells coexpress the serine proteases TMPRSS2 and TMPRSS4, which promote SARS-CoV-2 spike protein fusion and viral entry into enterocytes (6).

HIV infection in the GI tract results in rapid and massive $\mathrm{CD} 4^{+} \mathrm{T}$ cell depletion, with associated changes in the microbiome and elevated translocation of microbial products across the epithelial barrier. These 
events precipitate a so-called leaky gut syndrome, which is thought to be central to HIV pathology (10-13). The elevated systemic immune activation, inflammatory responses, and gut dysbiosis associated with this syndrome (14) may influence the overall type I interferon responses, and therefore compromise both local and systemic responses to SARS-CoV-2 infection, including responses within the lung mucosa (15). Multiple studies prior to the SARS-CoV-2 pandemic have demonstrated that HIV-induced systemic immune activation renders HIV-infected individuals vulnerable to airborne infections, such as tuberculosis $(16,17)$. Recent data suggest that SARS-CoV-2 and HIV coinfected individuals overall have a 2.1-fold increased risk of dying from COVID-19 (16), with a risk factor reaching more than 3.5 in viremic individuals with CD4 count below 200 cells $/ \mathrm{mm}^{3}$ (18). This is consistent with low nadir CD4 $4^{+} \mathrm{T}$ cell counts being associated with increased mortality in COVID-19 patients (19). Therefore, understanding the dynamics of SARS-CoV-2 infection in the gut of HIV-infected individuals is a pressing area of research.

Single-cell transcriptomic analysis from different tissues reported increased coexpression of $A C E 2$ and TMPRSS2 transcripts in the ileum of SHIV-infected nonhuman primates and within the lung of HIV-infected humans compared with uninfected controls (20), suggesting a potential higher risk of SARS-CoV-2 infection in HIV-infected individuals. One of the isoforms of $A C E 2$, encoded by exon 1c (dACE2), has recently been confirmed as an interferon-stimulating gene (ISG) and responds directly to type I interferons and SARS-CoV-2 infection in the small intestine (21). Therefore, type 1 interferon stimulation and altered regulation of $A C E 2$ could be exploited by SARS-CoV-2 to enhance infection (20). However, no human studies have determined the impact of HIV infection on SARS-CoV-2 entry receptors in the gut, or on the transcriptional landscape of the gut enterocytes expressing them.

To explore the potential impact of HIV infection on gut epithelial cells susceptible to SARS-CoV-2 infection, we investigated the gene expression profile of ACE2, TMPRSS2, and TMPRSS4 using single-cell RNA-Seq (scRNA-Seq) data sets from human SARS-CoV-2-uninfected tonsil, liver, lymph node, duodenum, and blood, as well as a published human lung scRNA-Seq data set (20). All study participants were from clinics in KwaZulu-Natal, South Africa, recruited within extremely high HIV-1-endemic areas. Our data show that $A C E 2$ was associated with interferon response genes in HIV-uninfected individuals, supporting the hypothesis that $A C E 2$ expression is linked with interferon signaling within gut enterocytes. In addition, and as expected, we show that HIV infection itself drove a strong interferon signaling response in these cells. Surprisingly, however, HIV infection was associated with a downregulation of $A C E 2$ expression in all cell types studied. These data suggest that, although ACE2 is associated with interferon signaling genes, the isoforms detected here are differentially regulated from canonical interferon signaling genes. The reduction in potential target cells may affect infectability, propagation of infection of SARS-CoV-2 in HIV-infected individuals, and local and systemic immunity beyond the gut, such as the lung mucosa (15). Nevertheless, using gut biopsies from coinfected individuals, we observed the presence of SARS-CoV-2 and HIV proteins in gut enterocytes, indicating this compartment remains vulnerable to infection.

\section{Results}

$S A R S-C o V-2$ host entry receptors are enriched in the human small intestine. SARS-CoV-2 infection of human cells requires surface expression of the primary receptor ACE2 and one of the coreceptors TMPRSS2 (22) or TMPRSS4 $(3,6)$. scRNA-Seq analysis showed that ACE2 expression is primarily restricted to type II pneumocytes in the lung, gut absorptive enterocytes, and goblet secretory cells of the nasal mucosa (20). In this study, we applied high-throughput scRNA-Seq $(23,24)$ to profile distinct human tissue samples (Figure $1 \mathrm{~A}$ ) and analyzed 32,381 cells from blood, tonsil, lung, duodenum, and mesenteric lymph node (lung data set has been previously described in ref. 20) (Figure 1B), for expression of genes encoding ACE2, TMPRSS2, and TMPRSS4. We identified 18 distinct cellular subclusters in this data set (Supplemental Figure 1A; supplemental material available online with this article; https://doi.org/10.1172/jci.insight.148920DS1), with the majority of cells expressing ACE2, TMPRSS2, and TMPRSS4 located within duodenum and to a lesser extent in lung tissue and with no expression in the other tissues analyzed (Figure 1C). We next used fluorescence immunohistochemistry to costain epithelial cells (EpCAM) for ACE2 and TMPRSS2 and found distinct in situ expression patterns between the small and large intestine (Figure 1D and Supplemental Figure 1B). The duodenum tissue expressed high levels of $A C E 2$ facing outward toward the lumen side, whereas the colon tissue was characterized by ACE2 expression at the bottom half of the crypt. TMPRSS2 was expressed in most of the epithelial cells and was coexpressed with $A C E 2$ in both duodenum and colon tissue. We found no quantitative differences in protein expression levels between both compartments for 
A

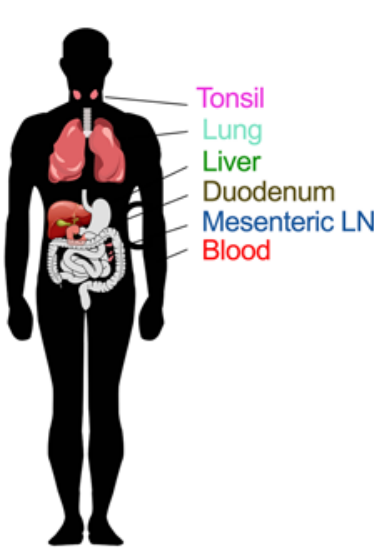

Seq-Well

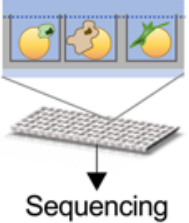

Library

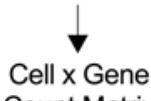

Count Matrix
Dimensionality Reduction, Clustering $\longrightarrow$ Cell Type Calling $\longrightarrow$
B

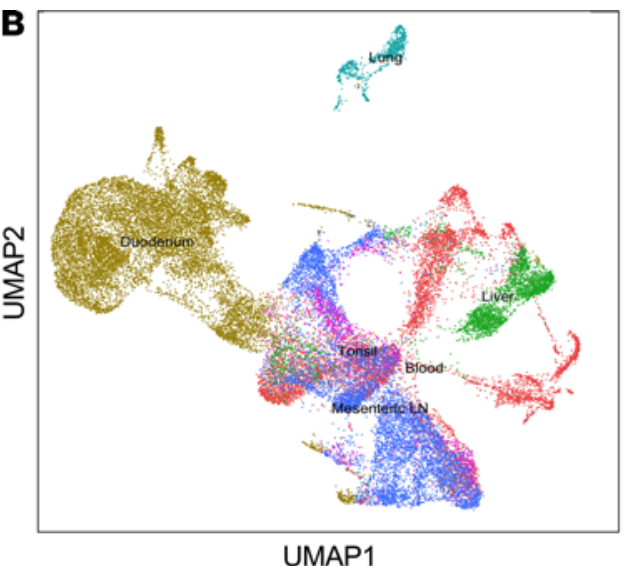

C

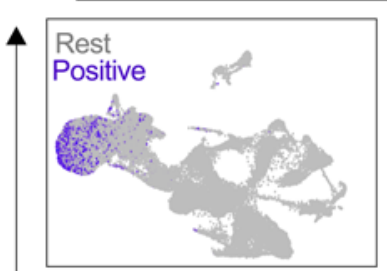

ACE2

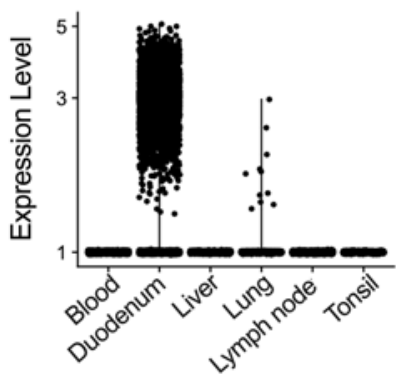

TMPRSS2
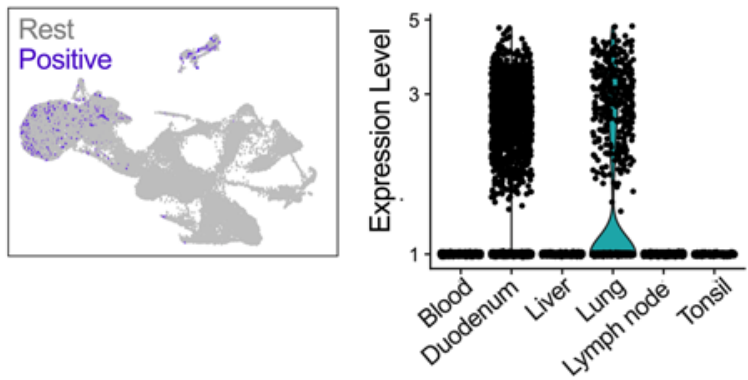

TMPRSS4

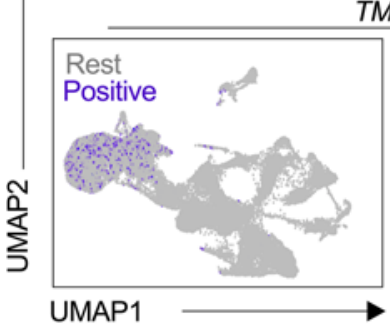

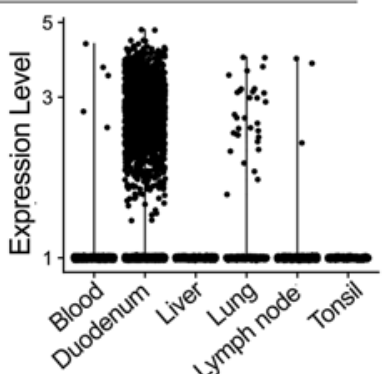

D
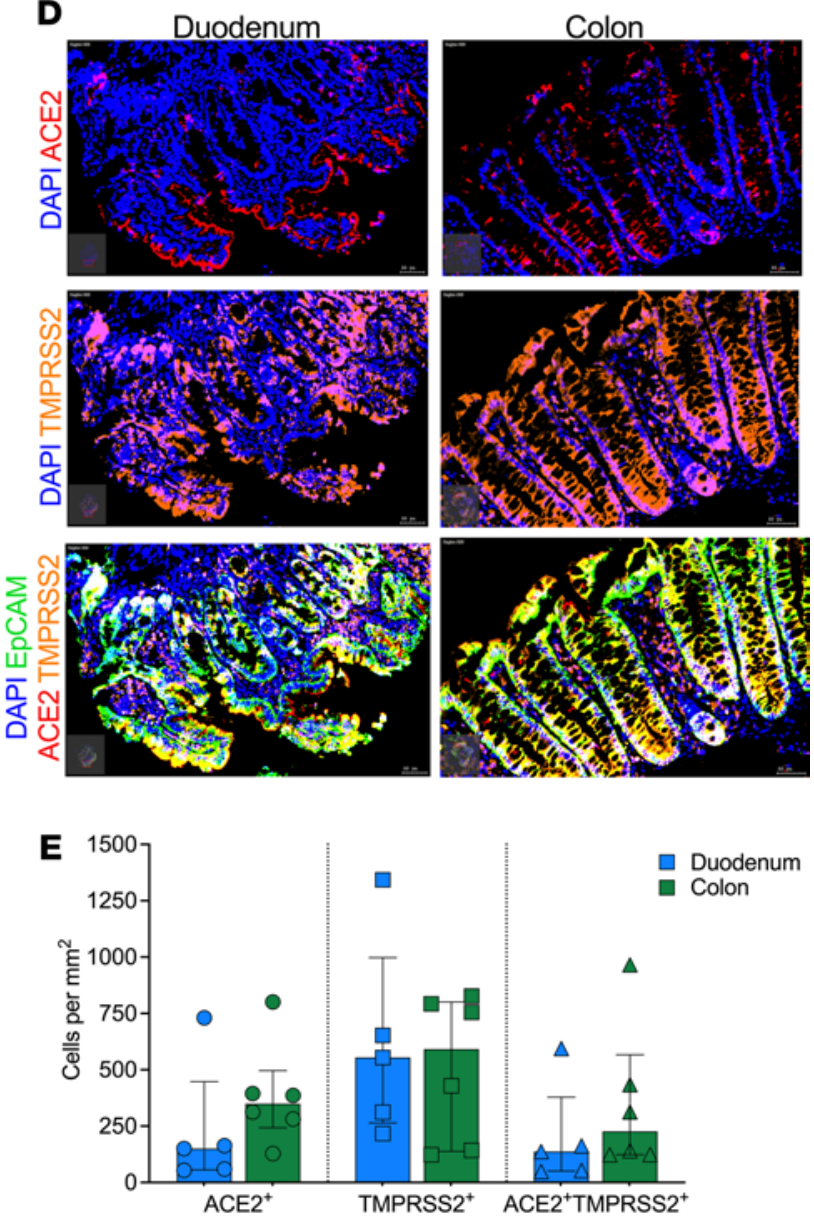

Figure 1. SARS-CoV-2 putative target cells are enriched in the human duodenum. (A) Schematic of protocol for isolation of different tissues for scRNA-Seq using Seq-Well S $^{3}$, to identify cell types. (B) Uniform manifold approximation and projection (UMAP) of 32,381 cells colored by tissue source. (C) Left: UMAP of epithelial cells showing expression of ACE2 (top), TMPRSS2 (middle), and TMPRSS4 (bottom) among all tissue sources from human donors. Color coding is as follows: purple, RNA positive; gray, RNA negative. Right: Corresponding violin plots of expression values for ACE2 (top), TMPRSS2 (middle), and TMPRSS4 (bottom). (D) Representative fluorescence immunohistochemistry image of gut tissue showing ACE2 (red), TMPRSS2 (orange), EpCAM (green), and DAPI (blue) of duodenum and colon. Bars: $20 \mu \mathrm{m}$ for all images. (E) Quantification of ACE2 and TMPRSS2 proportion of total cells stained with EpCAM. Data shown as median \pm SD.

ACE2 and TMPRSS2 (Supplemental Table 1 and Figure 1E). High SARS-CoV-2 entry receptor expression in the small intestine is consistent with recent reports $(2,5,6,20)$ and with detection of SARS-CoV-2 nucleocapsid in the small intestine of infected individuals (3). Locational differences in $A C E 2$ expression between the duodenum and colon may be linked to the physiology of $A C E 2$ in these compartments, such as 
Table 1. Characteristics of study participants $(n=24)$

\begin{tabular}{|c|c|c|c|c|}
\hline & \multicolumn{3}{|c|}{ HIV $^{-}$} & \multirow{2}{*}{$\begin{array}{c}\text { HIV+ }^{+} \mathbf{A R T}^{+} \\
{ }^{\mathrm{B}} \text { Duo }(n=6)\end{array}$} \\
\hline & ${ }^{\mathrm{A}}$ Duo $(n=7)$ & ${ }^{A}$ Colon $(n=7)$ & ${ }^{\mathrm{B}}$ Duo $(n=4)$ & \\
\hline \multicolumn{5}{|l|}{ Demographics } \\
\hline Age in years, median (IQR) & $43(33-53)$ & $46.5(35-53)$ & $43.5(32-54)$ & $49(36.25-53.50)$ \\
\hline \multicolumn{5}{|l|}{ Sex, $n(\%)$ median (IQR) } \\
\hline Female, 19 (79) & $43(27-54)$ & $46.5(22-52)$ & $43.5(32-54)$ & $49(36-54)$ \\
\hline Male, 5 (21) & $39(33-44)$ & $50(42-57)$ & NA & NA \\
\hline \multicolumn{5}{|l|}{${ }^{\circ}$ Ethnicity, $n(\%)$} \\
\hline Black, 14 (63) & $3(43)$ & $3(43)$ & $3(75)$ & $5(83)$ \\
\hline Colored, $1(4)$ & NA & $1(14)$ & NA & NA \\
\hline Indian, 8 (33) & $4(57)$ & $3(43)$ & $1(25)$ & $1(17)$ \\
\hline White, 1 (4) & NA & NA & $1(25)$ & NA \\
\hline CD4 count (cells/ $\mu \mathrm{L}$ ), median (IQR) & ND & ND & ND & $454(378-504)$ \\
\hline Viral load (copies/mL) & NA & NA & NA & $<20$ \\
\hline
\end{tabular}

regulation of intestinal inflammation and amino acid homeostasis $(7,8)$, and may influence SARS-CoV-2 infection dynamics in each compartment.

ACE2 expression is dominated by absorptive enterocytes. Next, focusing on the small intestine only, for which most samples were available, we evaluated the expression of ACE2, TMPRSS2, TMPRSS4, and other transmembrane serine proteases in duodenal cellular subsets using scRNA-Seq data from 4 HIV-uninfected female donors (Table 1 and Supplemental Table 1). To assign cellular identity, we performed variable gene selection, performed dimensionality reduction by UMAP with graph-based clustering (Figure 2A), created a cells-by-genes expression matrix (Figure 2B), and identified 15 distinct major cell clusters from 13,056 duodenum cells. Cell cluster identities were assigned based on the most highly differentially expressed genes (DEGs) for each cluster (Figure 2B and Supplemental Table 2A). This approach identified absorptive enterocytes as the predominant $A C E 2$-expressing cell type, defined by expression of APOA1 and APOA4 $\left(41.3 \% A C E 2^{+}\right.$, FDR-adjusted $\left.P=1.01 \times 10^{-227}\right)$ (Figure $\left.2 \mathrm{~B}\right)$. These cells also more frequently expressed TMPRSS2 and TMPRSS4 than any of the other subsets (Figure 2C and Supplemental Figure 2A). The majority of absorptive enterocytes also expressed ST14 and TMPRSS15 and to a much lesser extent TMPRSS3 and TMPRSS6 (Supplemental Figure 2B). There is no known role for these other serine proteases in SARS-CoV-2 infection, but it does imply the importance of this family of molecules to the biology of absorptive enterocytes (25). Focusing on epithelial cells, we consistently identified absorptive enterocytes as the main source of ACE2, TMPRSS2, and TMPRSS4 gene expression (Supplemental Figure 3, A-D) and SARS-CoV-2 putative target cells due to coexpression of these markers: $A C E 2^{+}$TMPRSS2 $2^{+}(412$ cells, $12 \%$ of all epithelial cells), $A C E 2^{+} T M P R S S 4^{+}$subset (392 cells, $11 \%$ of all epithelial cells), and a smaller subset coexpressing all 3 genes $A C E 2^{+} T M P R S S 2^{+} T_{M P R S S} 4^{+}$(154 cells, 5\%; Supplemental Figure $3 \mathrm{E}$ ). Using a Monocle single-cell trajectory analysis, we uncovered a clear potential differentiation trajectory from "intestinal stem cell-like" cells ( $L G R 5, C D 44, E P H B 2)$, through transit amplifying cells (OLFM4) and Paneth cells (DEFA5, DEFA6), to the dominant absorptive enterocyte clusters (APOA1, APOA4) enriched for putative SARS-CoV-2-susceptible cells (Supplemental Figure 4), suggesting that terminal differentiated cells are likely to be the most susceptible to infection. Thus, cellular differentiation was linked to SARSCoV-2 entry receptor expression. Overall, these observations are in line with previous reports indicating that TMPRSS2 and TMPRSS4 are important coreceptors for SARS-CoV-2 fusion and entry into $A C E 2^{+}$ differentiated enterocytes in the human duodenum and small intestine $(6,26)$.

$A C E 2$ expression is associated with ISGs. Next, we compared $A C E 2$-expressing $\left(A C E 2^{+}\right)$with nonexpressing $\left(A C E 2^{-}\right)$cells and identified 381 DEGs $(P<0.05)$ (Supplemental Table $\left.2 \mathrm{~B}\right)$. The majority of these genes were upregulated together with APOB and APOA4, confirming that ACE2 expression is associated with absorptive enterocytes (Figure 3A). In addition, these DEGs included a range of known interferon response genes, such as ISG20, which interferes with viral replication (27); STAT6, which is important for immune signals emanating from interleukin-4 receptors (28); TNFSF10, a proapoptotic cytokine $(29,30)$; TNFRSF1A involved in TNF- $\alpha$ signaling; and the proinflammatory cytokine $I L-32$, which is involved in the pathogenesis 
A
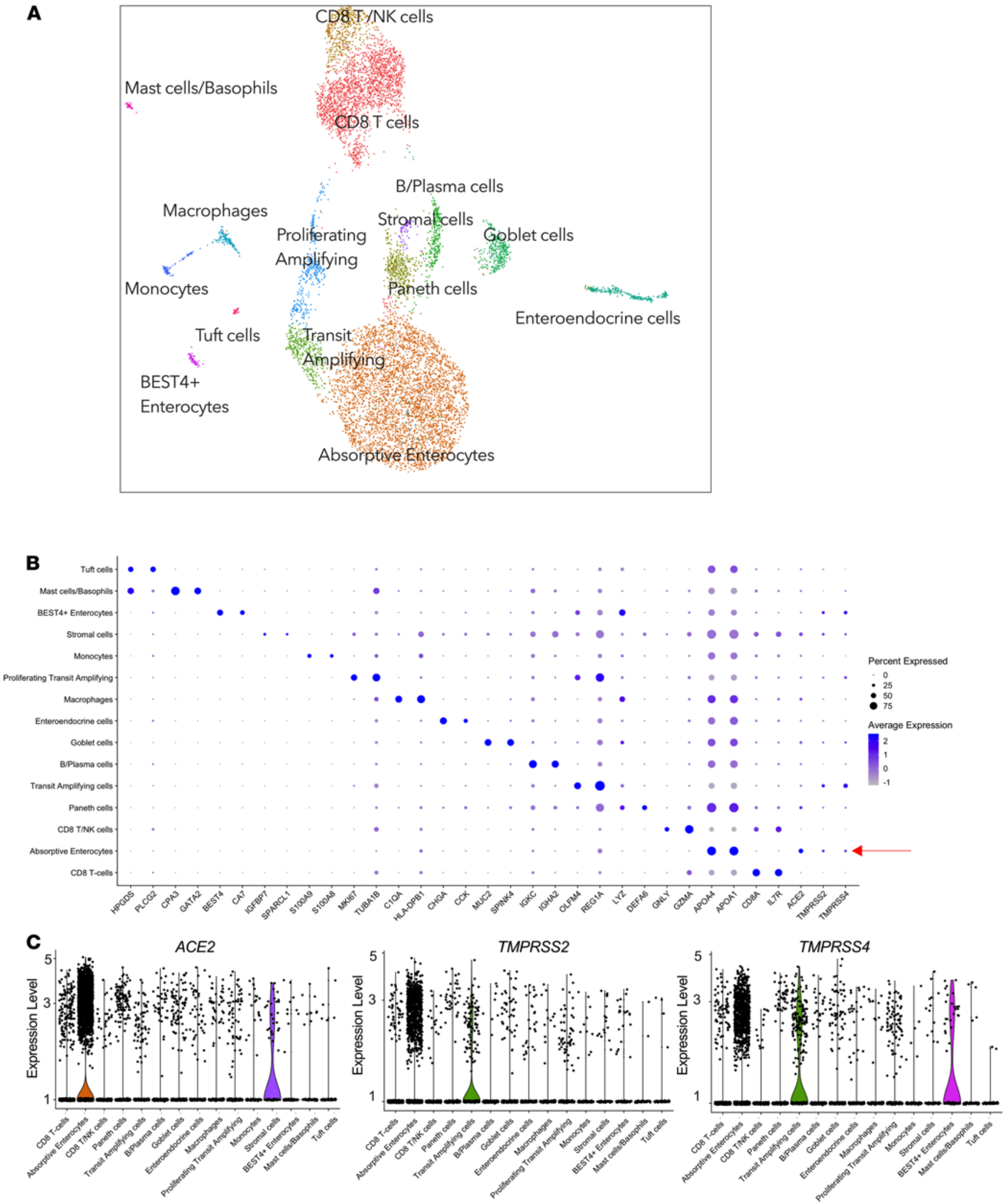

Figure 2. ACE2 expression is enriched in absorptive enterocytes. (A) UMAP of 13,056 cells from endoscopic pinch biopsies, colored by cell type. (B) Dot plot of 2 defining genes for each cell type and ACE2, TMPRSS2, and TMPRSS4. Dot size represents fraction of cells within cell type expressing a given gene, and color intensity represents binned count-based expression amounts $\left(\log _{\text {saled } y m+1+1}\right)$ among expressing cells. Red arrow indicates cell type with largest proportion of ACE2 ${ }^{+}$TMPRSS2 ${ }^{+}$TMPRSS4+ cells; full results can be found in Supplemental Table 2A. (C) Expression of ACE2 (left), TMPRSS2 (middle), and TMPRSS4 (right) among all subsets from duodenum. UMI, unique molecular identifier. 
and progression of a number of inflammatory disorders (31) (Figure 3B). In support of the cell subsetting approach, pathway analysis of $A C E 2^{+}$cells was consistent with functional absorptive cells, including pathways involved in transport of small molecules, mineral absorption, intestinal absorption, bile secretion, etc. (Figure 3C). Further subsetting on $A C E 2^{+} T M P R S S 2^{+}$cells as putative SARS-CoV-2 targets and comparison with the remaining epithelial cells (Supplemental Table 2C) showed significant upregulation of IFNGRI within $A C E 2^{+}$TMPRSS2 $^{+}$cells (Supplemental Table 2C), further supporting the expression of ISG signatures in susceptible target cells. Analysis of potential upstream drivers of the ACE2-associated DEGs identified canonical ISG genes, such as STAT3, IRF2, and IRF4 (Figure 3D and Supplemental Table 2D). These data show that epithelial cells, and particularly the absorptive enterocytes, are enriched for ACE2, TMPRSS2, and TMPRSS4 expression and that ACE2 expression in these cells is upregulated in conjunction with known ISGs.

HIV infection drives interferon response in gut absorptive enterocytes. HIV infection depletes $\mathrm{CD} 4^{+} \mathrm{T}$ cells in the gut and has a major impact on the barrier integrity $(10,12)$, and the intestinal epithelium is dominated by absorptive enterocytes with high expression levels of SARS-CoV-2 entry receptors compared with other epithelial subsets (see Supplemental Figure 3 and Supplemental Table 3A). Therefore, we next determined the influence of HIV infection on potential SARS-CoV-2 target cells, by comparing the gene expression of cells from duodenal tissues collected from individuals with chronic HIV infection on long-term antiretroviral therapy (ART) and HIV-uninfected participants. We found 160 DEGs (134 upregulated and 26 downregulated; Figure 4A and Supplemental Table 3B). Consistent with the gut mucosal inflammation associated with HIV (32), these included strong upregulation of numerous canonical ISG and antiviral genes, including ISG15, IFI6, LY6E, IFITM3, IFI27, MX1, and IRF7 (Figure 4B and Supplemental Table 3B). In contrast to earlier studies (20), and despite the observed interferon signaling, $A C E 2$ expression was significantly reduced in enterocytes isolated from subjects with treated chronic HIV infection (Figure 4B), suggesting that $A C E 2$ expression itself may not directly act as an ISG (21). Pathway analysis of DEGs in absorptive enterocytes showed that HIV infection was associated with a profound upregulation of interferon signaling (Figure 4C). Analysis of potential upstream drivers of these DEGs indicated type I interferon and multiple interferon response factors, consistent with a constitutive antiviral response program within absorptive enterocytes that was induced by HIV infection despite ART (Figure 4D and Supplemental Table 3C). Finally, subsetting on the remaining $A C E 2$-expressing epithelial cells only showed a consistent strong upregulation of these canonical ISGs in HIV-infected subjects (Supplemental Figure 5, A and B, and Supplemental Table 3D). Taken together, these data show that, despite suppression of plasma viremia, HIV infection induces a strong interferon antiviral response in gut enterocytes in general and also within ACE2-expressing putative SARS-CoV-2 target cells. However, this does not drive ACE2 expression itself, which is reduced in HIV-infected individuals.

Reduced numbers of ACE2-expressing absorptive enterocytes and SARS-CoV-2 target cells in HIV-infected individuals. Having observed an unexpected downregulation of $A C E 2$ in HIV-infected subjects, we quantified the number of epithelial cells susceptible to SARS-CoV-2 infection and compared the relative frequencies of absorptive enterocytes, goblet cells, and transit-amplifying cells (see Figure 2) between HIV-infected and uninfected individuals. Overall, the numbers of ACE2- and TMPRSS2-expressing absorptive enterocytes were significantly reduced in HIV infection, while TMPRSS4 was unaffected (Figure 5A). The same trend was observed for goblet cells but not for transit-amplifying cells (Figure 5, B and C). When we analyzed absorptive enterocytes expressing 2 or more entry receptors, we found a similar significant reduction of all combinations of SARS-CoV-2 putative target cells, with the same trend for goblet cells but not for transit-amplifying cells (Supplemental Figure 6). Thus, chronic HIV infection appears to reduce the total frequency of SARS-CoV-2 putative target cells within the small intestine.

Abundant SARS-CoV-2 detection in small and large intestine irrespective of HIV coinfection. Finally, to determine if HIV-associated loss of SARS-CoV-2 target cells and upregulation of antiviral genes prevented infection of the GI tract, we collected gut tissue from confirmed SARS-CoV-2 antigen PCR-positive participants with and without HIV infection. From a duodenum biopsy obtained from a SARS-CoV-2 and HIV coinfected individual on ART and with undetectable plasma HIV viremia (Supplemental Table 1), we identified abundant expression of SARS-CoV-2 nucleocapsid protein (NP) within the epithelial layer and colocalized with that of ACE2 entry receptor (Figure 6A). We found small, but detectable, levels of HIV-p24 protein in the same area but in cells that did not express ACE2, consistent with distinct viral entry receptor usage between SARS-CoV-2 and HIV infection. We repeated this staining in a pre-pandemic control sample and observed no SARS-CoV-2 NP staining (Figure 6B). Histology of colon samples collected from an HIV-uninfected SARSCoV-2 PCR-positive donor also revealed abundant SARS-CoV-2 NP expression within the epithelial tissue 
A

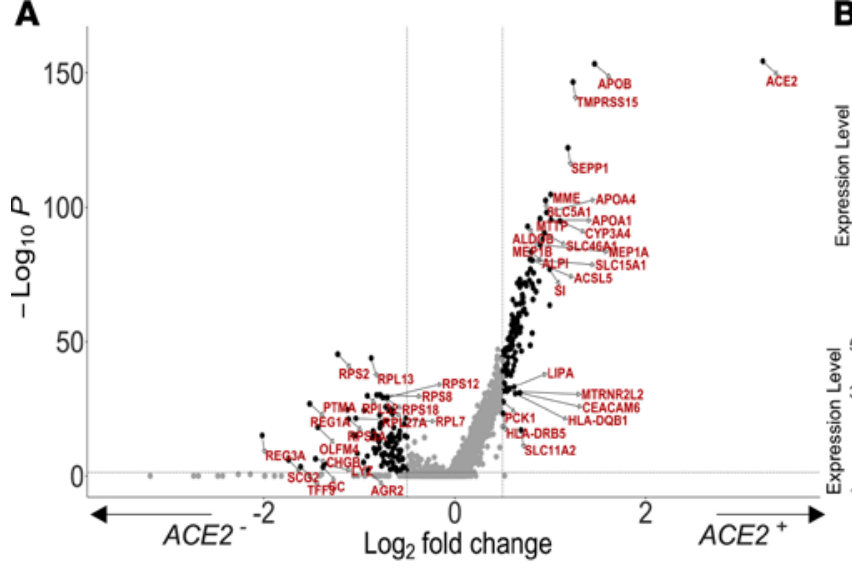

B

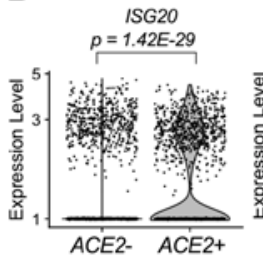

IL.32

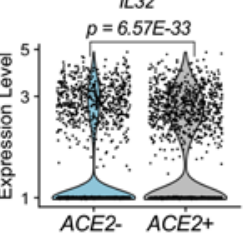

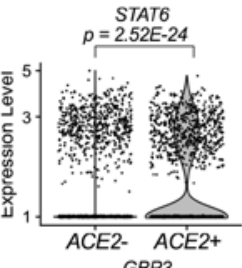

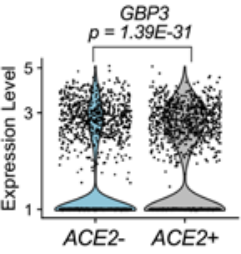

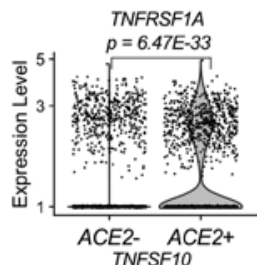

TNFSF10
$P=4.99 E-32$

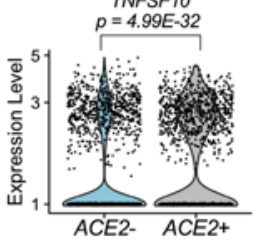

C

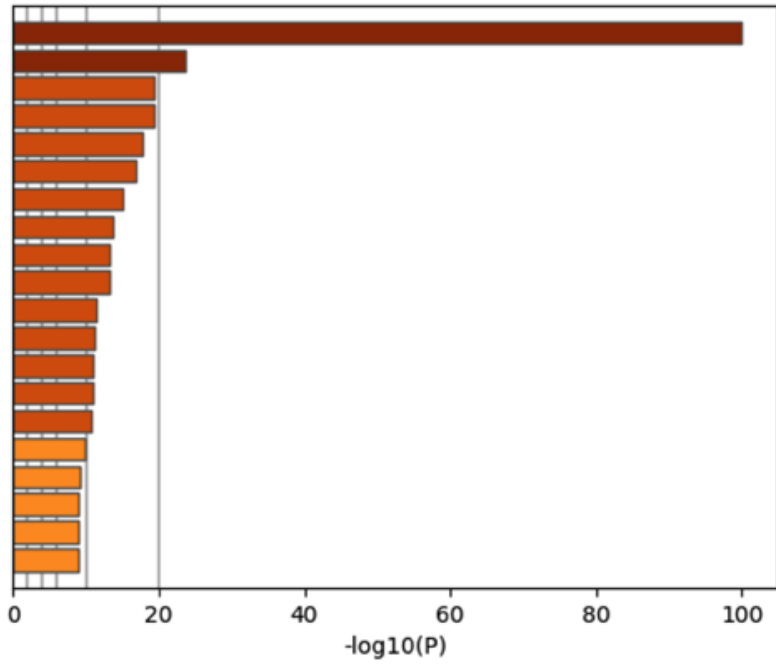

CORUM:306: Ribosome, cytoplasmic

R-HSA-382551: Transport of small molecules

R-HSA-6798695: Neutrophil degranulation

GO:0032787: monocarboxylic acid metabolic process

ko04978: Mineral absorption

GO:0042255: ribosome assembly

GO:0050892: intestinal absorption

GO:0042445: hormone metabolic process

ko04612: Antigen processing and presentation

GO:0015711: organic anion transport

R-HSA-556833: Metabolism of lipids

hsa03320: PPAR signaling pathway

R-HSA-211859: Biological oxidations

ko04974: Protein digestion and absorption

GO:0009636: response to toxic substance

GO:0008202: steroid metabolic process

CORUM:5266: TNF-alpha/NF-kappa B signaling complex 6

ko04976: Bile secretion

GO:0050821: protein stabilization

GO:0042737: drug catabolic process

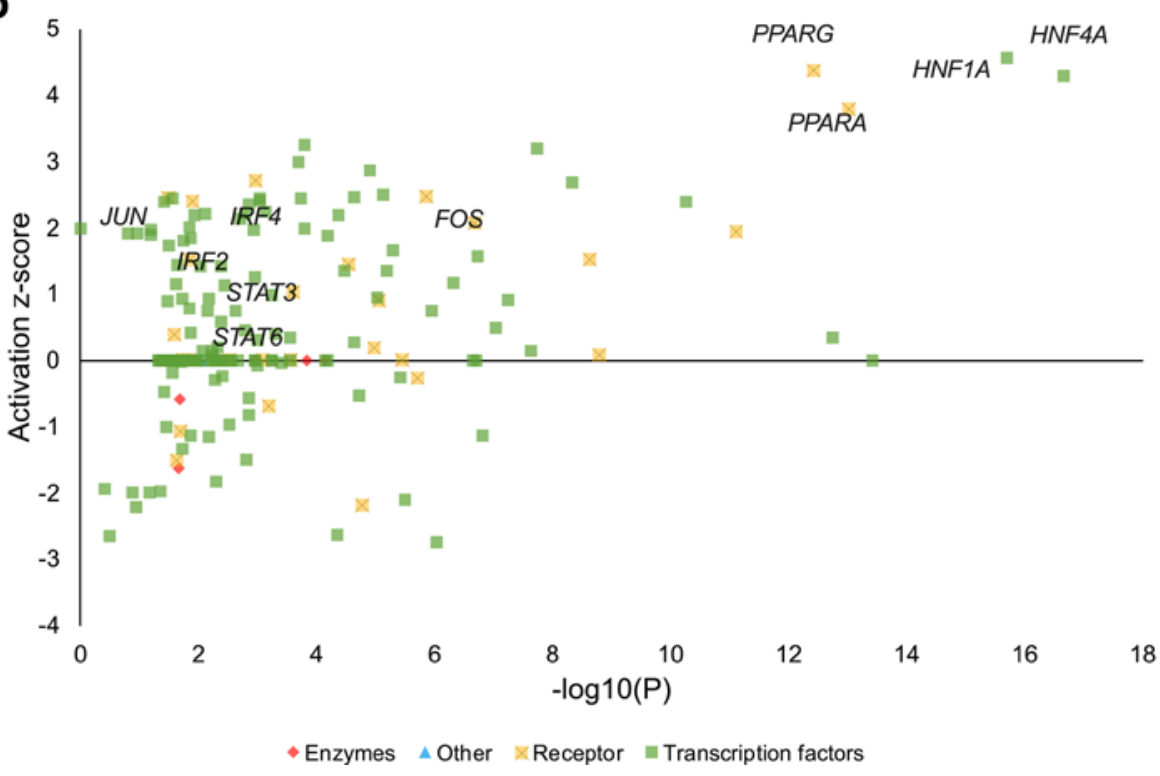

Figure 3. ACE2-expressing absorptive enterocytes are linked to ISGs and functional absorptive pathways. (A) Volcano plot of DEGs (Supplemental Table 2B) within epithelial cells from HIV-uninfected individuals $(n=4)$ highlighting genes with more than 0.5 fold change and adjusted $P<5.0 \times 10^{8}$. (B) Violin plots of genes differentially expressed among $A C E 2^{+}$and $A C E 2^{-}$epithelial cells, FDR-adjusted $P<0.05$; full results can be found in Supplemental Table $2 B$. (C) GO BP enrichment analysis of the DEGs from epithelial cell analysis upregulated in $A C E 2^{+}$compared with $A C E 2^{-}$. $P$ value was derived by a hypergeometric test. (D) Selected upstream drivers of pathways shown in C from DEGs in Supplemental Table 2D. GO, Gene Ontology; BP, biological pathway. 
overlapping with ACE2 (Figure 6C). These data confirm the presence of high levels of SARS-CoV-2 virus production in the GI tract (3) and show it is likely to occur in HIV-infected individuals despite upregulation of antiviral immunity and a loss of putative target cells in the small intestine.

\section{Discussion}

In this study we performed single-cell transcriptomic profiling across different human tissue sites and identified high expression of SARS-CoV-2 entry receptors within absorptive enterocytes from the small intestine that we confirmed by in situ protein staining. We detected overlapping expression of SARS-CoV-2 NP and ACE2 within both the small and large intestine of SARS-CoV-2-infected individuals, confirming the infectability of these cells in vivo. ACE2, TMPRSS2, and TMPRSS4 expression was highest in the duodenum followed by the lung, with little or no expression detected in the tonsil, liver, lymph node, and blood, consistent with published studies $(20,25,33,34)$. We found that ACE2 protein expression was restricted to the luminal region of the enterocytes in the duodenum, whereas in the colon, ACE2 was located closer to the crypt base $(7,8)$. This distinct location may be explained by differences in the physiological processes within these compartments where luminal duodenal ACE2 is reported to be important in amino acid transport and protein synthesis $(35,36)$, consistent with our pathway analysis of $A C E 2$-expressing duodenal epithelial subsets (see Figure 3C).

In HIV-uninfected subjects, $A C E 2$ expression in the small intestine was associated with genes involved in interferon signaling, in agreement with recent observations and experimental data demonstrating upregulation of ACE2 in response to interferon signaling (20). However, more recently investigators have established that it is the truncated isoform of ACE2, $d A C E 2$, that is most likely to act as an ISG and not ACE2. This study found that $d A C E 2$ was directly upregulated by both interferon stimulation and SARS-CoV-2 infection within human intestinal organoid cells, but the full-length $A C E 2$ was not (21). Importantly, $d A C E 2$ does not function as a SARS-CoV-2 entry receptor (37). In the future, it would be interesting to also sequence the gut virome of these individuals to determine how this may contribute to interferon signaling within the intestinal mucosa. The transcriptomic profile of enterocytes from chronic HIV-infected individuals was characterized by a strong interferon signaling pathway that included upregulation of canonical ISGs such as ISG15 and IFI27 predicted to be driven by type I and II interferons. These data highlight the impact from HIV infection on the small intestine and contribute to understanding of mechanisms underlying the functional consequences in gut barrier integrity and overall pathology (10-13). The reduced frequency of $A C E 2$-expressing cells in the intestinal mucosa was therefore unexpected. Both ACE2 and canonical ISGs' (STAT1 and IFI6) levels remained elevated in absorptive enterocytes from nonhuman primates (NHPs) with treated SIV infection (20). Indeed, IFI6 was also highly upregulated in HIV-infected subjects in our cohort (Figure 3B). Why ACE2-expressing cells were reduced is not clear from this study. Changes in the microbiome, however, have recently been shown to alter ACE2 expression levels and disrupt the ACE/ACE2 axis, and HIV is known to cause gut dysbiosis (38, 39). It will therefore be interesting to investigate the link between HIV infection, altered microbiomes, and ACE2 expression levels in the gut of HIV-infected individuals. The Monocle lineage analysis conducted here suggests that ACE2 expression may be upregulated as enterocytes progress toward terminal differentiation. Therefore, a reduction of ACE2-expressing cells could result from interference in this process or in increased cell death of terminally differentiated ACE2-expressing cells. Individuals in this study were all women on long-term, fully suppressive ART, which may distinguish them somewhat from the experimentally infected NHPs, which were treated for 6 months. In addition, the impact of the gut microbiome on ACE2 expression in the small intestine, discussed above, may affect comparisons between experimental NHP studies and human cohorts $(38,39)$. We only used women for the transcriptional data in this study to avoid sex-biased gene expression, and therefore extending these gene expression profiles beyond women will require further validation. However, the persistent interferon signature in these women clearly implies that upstream drivers, such as type I and II interferons, have not diminished.

Although the implications for infectability of the gut mucosa for the SARS-CoV-2 virus remain unclear, our data from HIV and SARS-CoV-2 coinfected participants from whom we obtained ex vivo duodenum biopsies showed that SARS-CoV-2 infection certainly can occur in the gut of HIV-infected individuals. Whether HIV-infected individuals have longer SARS-CoV-2 sequelae from the gut (3) would be interesting to study in a large coinfected gut biopsy cohort, as would the impact of variant viruses, which may have different affinities for cell entry receptors $(40,41)$.

Although the literature is still emerging, in general, studies have observed that COVID-19 patients with controlled HIV coinfection and preserved $\mathrm{CD}^{+} \mathrm{T}$ cell counts have similar clinical trajectories to those without 
A
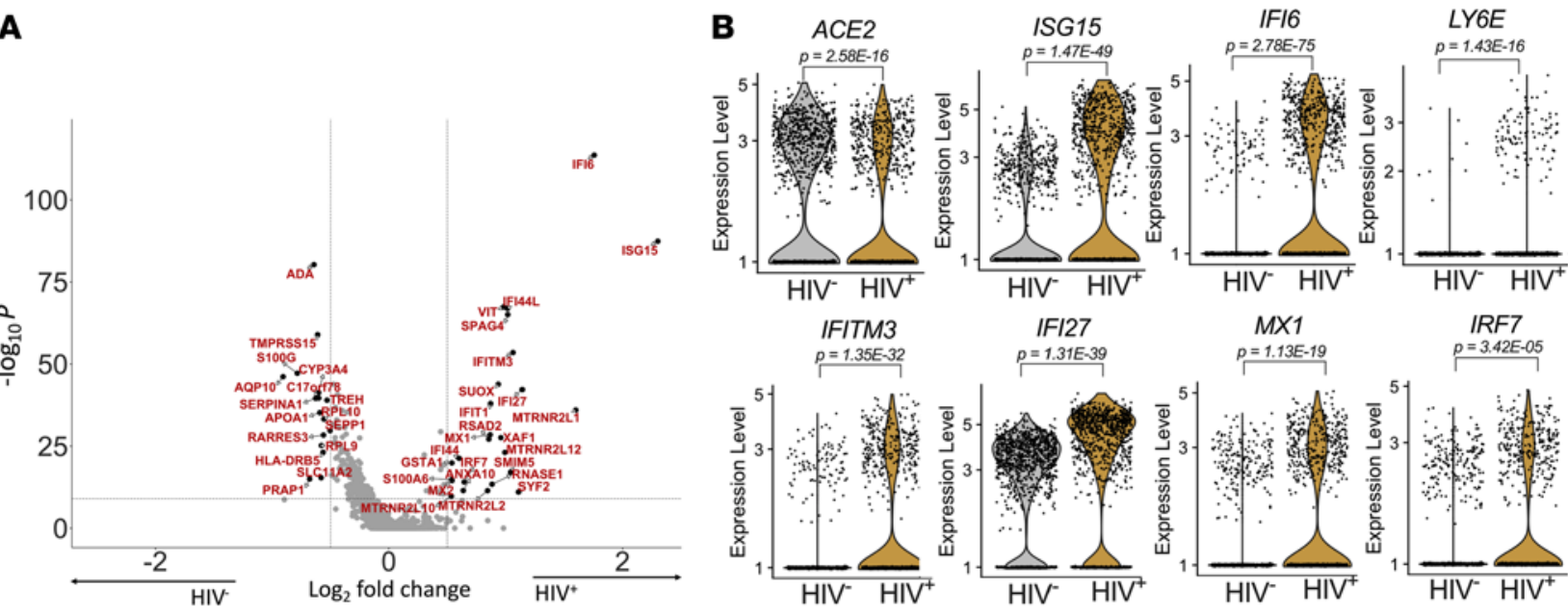

C

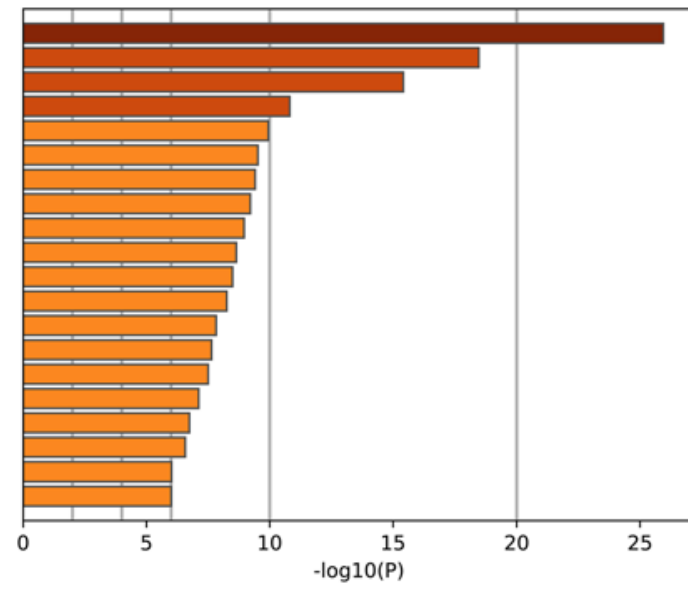

R-HSA-913531: Interferon Signaling GO:0034341: response to interferon-gamma CORUM:306: Ribosome, cytoplasmic

GO:0002697: regulation of immune effector process

GO:1900118: negative regulation of execution phase of apoptosis GO:0045088: regulation of innate immune response GO:0050892: intestinal absorption

GO:0002444: myeloid leukocyte mediated immunity

R-HSA-163200: Respiratory electron transport, ATP synthesis by chemiosmotic coupling GO:0010817: regulation of hormone levels

GO:0051186: cofactor metabolic process

GO:0032787: monocarboxylic acid metabolic process

GO:0007005: mitochondrion organization

GO:0050777: negative regulation of immune response

GO:0046718: viral entry into host cell

ko04978: Mineral absorption

ko04977: Vitamin digestion and absorption

GO:0002250: adaptive immune response

GO:0009617: response to bacterium

GO:0003073: regulation of systemic arterial blood pressure

D

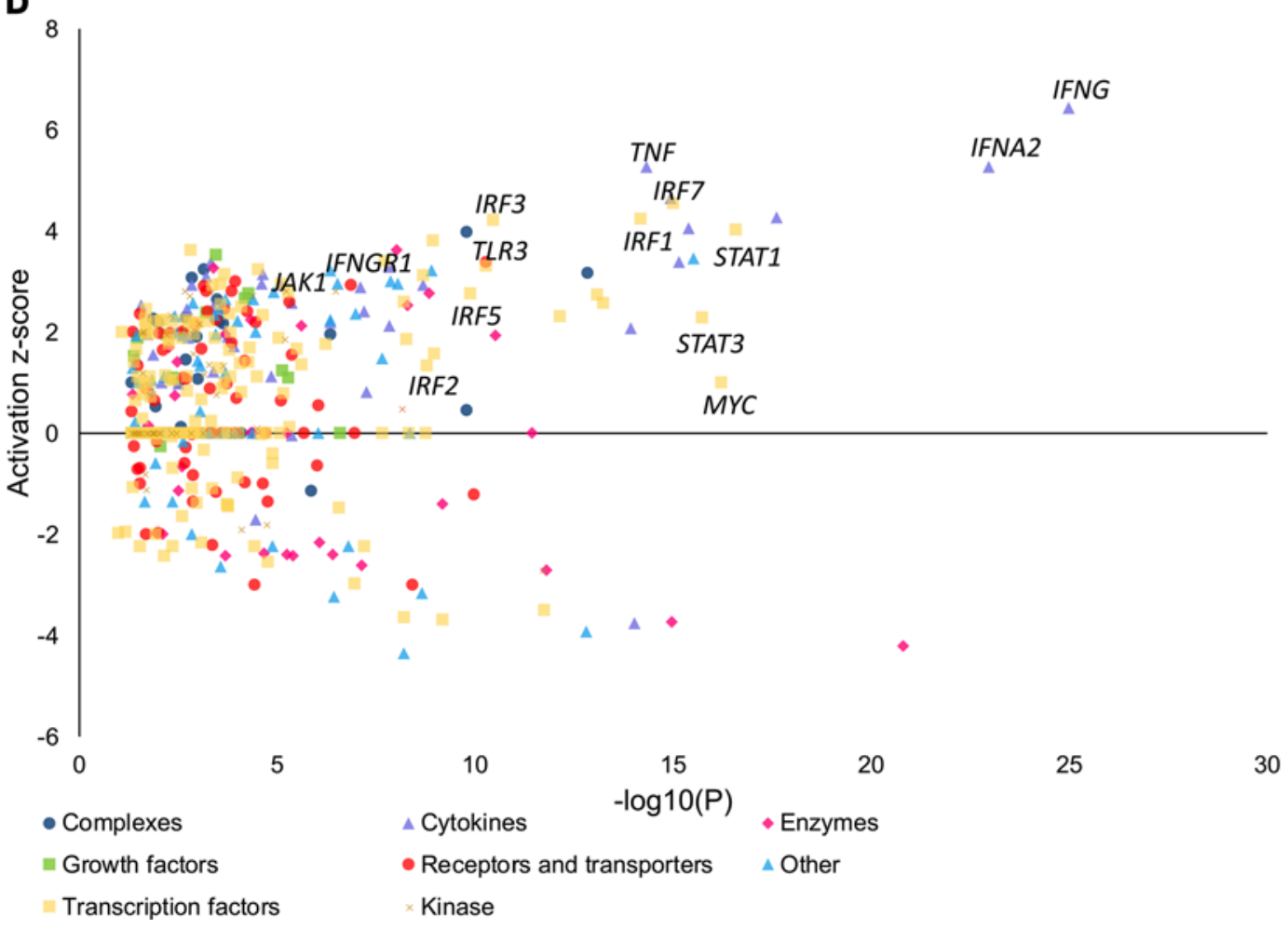


Figure 4. HIV infection downregulates ACE2 expression and drives interferon signaling in absorptive enterocytes. (A) Volcano plot of DEGs (Supplemental Table 3B) within absorptive enterocytes in HIV-infected and HIV-uninfected cells highlighting genes with more than 0.5 -fold change and adjusted $P<5.0 \times 10^{8}$. (B) Violin plots of expression of $A C E 2$ and interferon-responsive genes among absorptive enterocytes from HIV- $(n=4)$ and $\mathrm{HIV}^{+} A R T^{+}(n=5)$. (C) GO BP enrichment analysis of the DEGs of absorptive enterocytes upregulated in $\mathrm{HIV}^{-}(n=4)$ and $\mathrm{HIV}^{+} \mathrm{ART}^{+}(n=5) . P$ value was derived by a hypergeometric test. (D) Activation $z$ score of upstream drivers from DEGs shown in $\mathbf{A}$ and Supplemental Table 3D color-coded by their functional categories.

HIV infection $(18,42,43)$. By contrast, immune-compromised, HIV-infected individuals with CD4 ${ }^{+} \mathrm{T}$ cell counts below 200 cells $/ \mathrm{mm}^{3}$ are associated with increased COVID-19 disease severity and mortality $(16,18)$. Whether reduction of potential SARS-CoV-2 target cells in gut mucosa of HIV-infected subjects limits the additional effects of SARS-CoV-2 infection in this compartment warrants further studies. Further intestinal sampling from coinfected individuals is currently being sought to address this urgent question. Indeed, although the actual number of SARS-CoV-2 target cells was reduced, interferon signaling and pathways of metabolic and absorptive changes observed here in HIV-infected subjects prior to SARS-CoV-2 infection may still exacerbate disease or limit immunity in some individuals. Whether increased transmissibility of new SARS-CoV-2 variants, including beta and the recent dominant delta variants identified in high HIV prevalence populations in South Africa $(44,45)$, alter the GI tract-related symptoms and overall COVID-19 disease severity is also currently unknown. Finally, potentially altered induction of type I interferons by evolving SARS-CoV-2 variants could potentially also be linked to signaling within intestinal enterocytes and should be explored further.

\section{Methods}

Study participants. Patients presenting to the GI surgical unit of Inkosi Albert Luthuli Central Hospital were recruited into this study after they provided written informed consent. Tonsil, liver, gut lymph node, duodenum, and colon biopsies with participant-matched blood samples were obtained during surgical procedures. Clinical information, including HIV status and demographic details of these participants, was collected using a structured questionnaire. HIV status was confirmed using the Determine HIV 1/2 Set (Abbott Laboratories) and COBAS TaqMan HIV-1 Test (Roche).

Sample processing. Mononuclear cells were isolated from blood, tonsil, liver, lymph node, and pooled duodenum pinches to average over individual pinch variation. Blood was collected in BD vacutainers with sodium heparin. Peripheral blood mononuclear cells were isolated using the Ficoll-Histopaque 1077 (MilliporeSigma) density gradient centrifugation. Duodenum and colon pinch biopsies ( 2 to 4 pinches) were removed by the operating GI surgeon and transported to the laboratory in cold PBS ( $\mathrm{pH}$ 7.2). The PBS was decanted from the tubes containing the gut biopsies, which are about $5-8 \mathrm{~mm}$ in size, and they were incubated in epithelial strip buffer (PBS, 0.5 M EDTA, $1 \mathrm{M} \mathrm{DTT,} \mathrm{FBS,} \mathrm{and} \mathrm{penicillin/streptomycin)} \mathrm{in} \mathrm{a} 37^{\circ} \mathrm{C}$ water bath for 10 minutes, with occasional agitation. Thereafter, the epithelial strip buffer was removed, and the tissues were digested in a buffer containing collagenase-D $(0.5 \mathrm{mg} / \mathrm{mL}$; Roche) and DNase I (20 $\mu \mathrm{g} / \mathrm{mL}$; MilliporeSigma) for $30 \mathrm{~min}$ utes in a $37^{\circ} \mathrm{C}$ water bath with occasional agitation. Digested tissue was passed through a $70 \mu \mathrm{m}$ cell strainer to isolate the cells, and these cells were washed with PBS.

Single-cell RNA-Seq using Seq-Well $S^{3}$. After obtaining single-cell suspensions from fresh biopsies, we used the Seq-Well S ${ }^{3}$ platform. Full methods on implementation of this platform are described $(23,24)$. Briefly 15,000 cells in $200 \mathrm{~mL}$ RPMI with 10\% FBS were loaded onto a PDMS array preloaded with barcoded mRNA capture beads (ChemGenes) and settled by gravity into distinct wells. The loaded arrays were washed with PBS and sealed using a polycarbonate membrane with a pore size of $0.01 \mu \mathrm{m}$, which allows for exchange of buffers but retains biological molecules within each nanowell. Arrays were sealed in a dry $37^{\circ} \mathrm{C}$ oven for 40 minutes and submerged in a lysis buffer containing guanidium thiocyanate (MilliporeSigma), EDTA, 1\% $\beta$-mercaptoethanol, and sarkosyl (MilliporeSigma) for 20 minutes at room temperature. Arrays were transferred to hybridization buffer containing $\mathrm{NaCl}$ (Thermo Fisher Scientific) and supplemented with $8 \%$ (v/v) polyethylene glycol (PEG, MilliporeSigma) and agitated for 40 minutes at room temperature. mRNA capture beads with mRNA hybridized were collected from each Seq-Well array, and beads were resuspended in a master mix for reverse transcription containing Maxima H Minus Reverse Transcriptase (Thermo Fisher Scientific EP0753) and buffer, dNTPs, RNase inhibitor, a 50 template switch oligonucleotide, and PEG for 30 minutes at room temperature, and overnight at $52^{\circ} \mathrm{C}$ with end-over-end rotation. Exonuclease I treatment was used (New England Biolabs [NEB] M0293L) to remove excess primers. After exonuclease digestion, beadassociated cDNA was denatured for 5 minutes in $0.2 \mathrm{mM} \mathrm{NaOH}$ with end-over-end rotation. Next, beads were washed with TE buffer $+0.01 \%$ Tween-20, and second strand synthesis was carried out by resuspending 
A Absorptive enterocytes

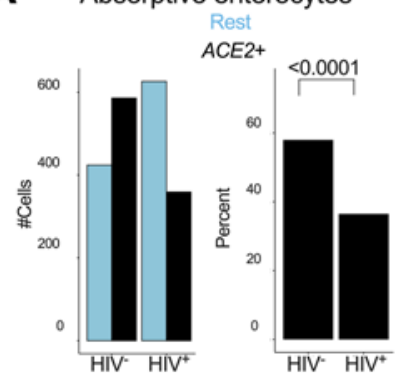

B Goblet cells
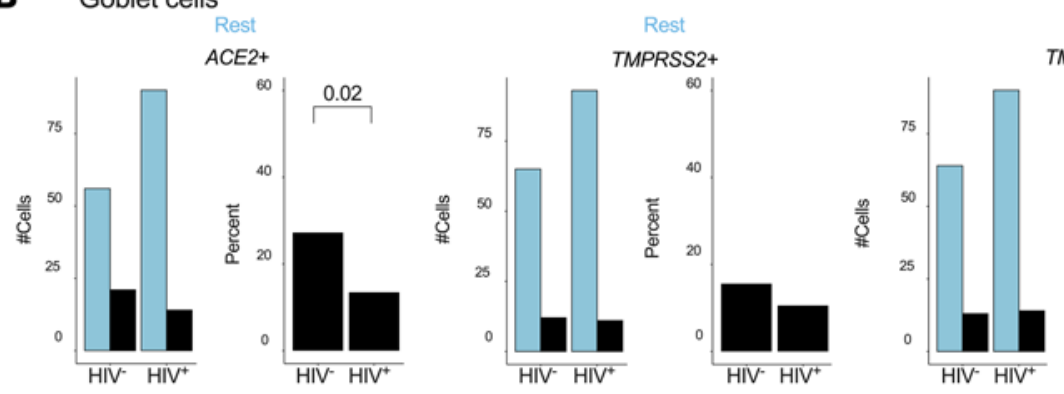

C Amplifying cells

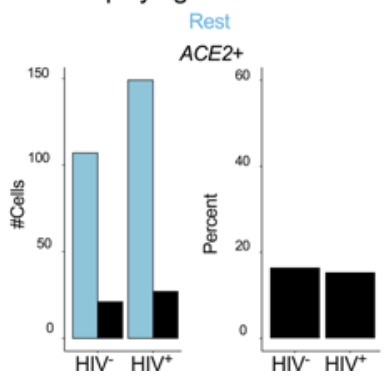

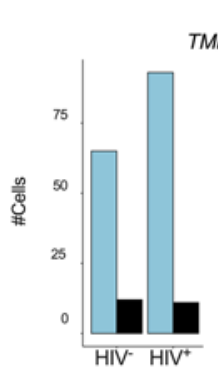
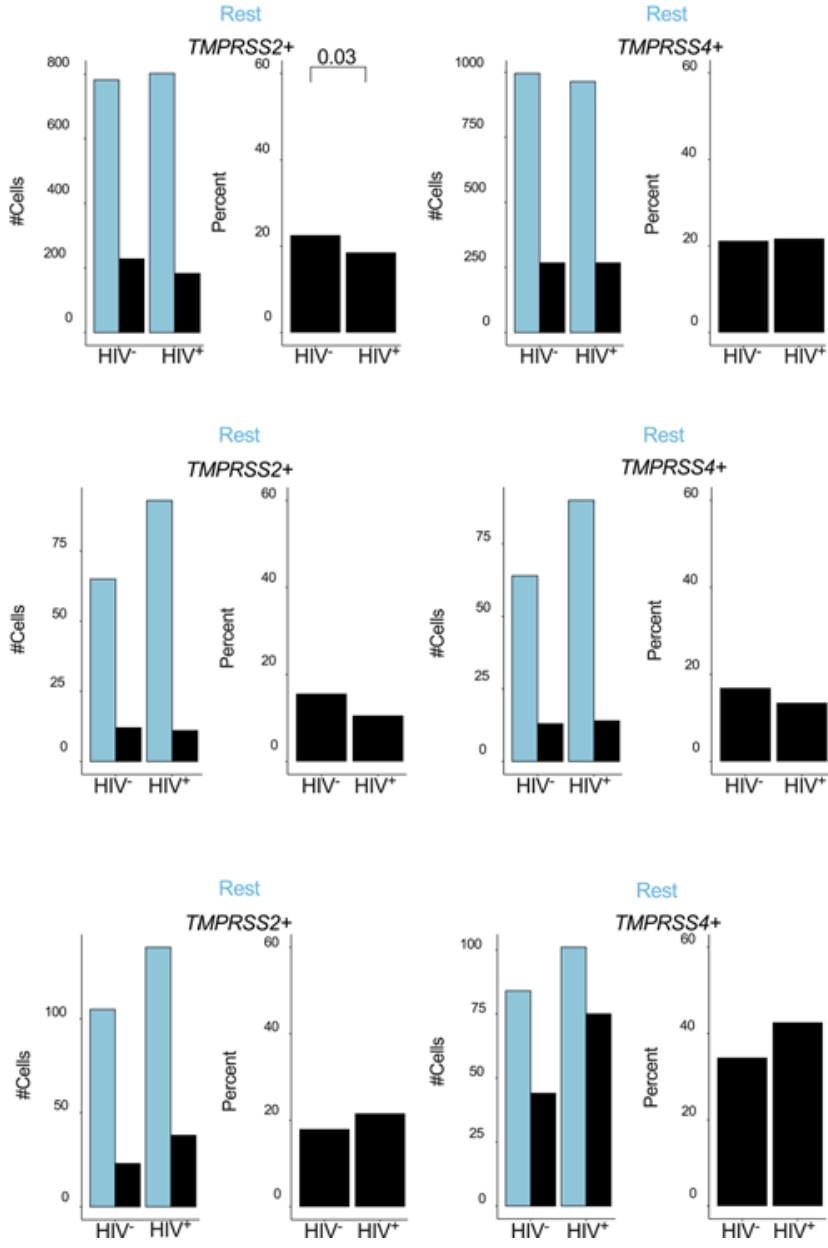

Figure 5. HIV infection reduces the frequency of SARS-CoV-2 putative target cells within the small intestine. (A) Actual number of absorptive enterocytes (left) and percentage expression (right) of ACE2, TMPRSS2, and TMPRSS4 by HIV status. (B) Number of goblet cells (left) and percentage (right) expressing ACE2, TMPRSS2, and TMPRSS4 by HIV status. (C) Number of transit-amplifying cells (left) and percentage (right) expressing ACE2, TMPRSS2, and TMPRSS4 by HIV status. $P$ values by Fisher's Exact Test. Rest, cells not expressing the indicated transcript.

beads in a master mix containing Klenow Fragment (NEB), dNTPs, PEG, and the dN-SMRT oligonucleotide to enable random priming off the beads. PCR amplification was carried out using KAPA HiFi PCR Mastermix (Kapa Biosystems KK2602) with 2.00 beads per $50 \mu \mathrm{L}$ reaction volume. After whole transcriptome amplification, libraries were pooled in sets of 6 (12.000 beads) and purified using Agencourt AMPure XP SPRI beads (Beckman Coulter, A63881) by a $0.6 \times$ volume ratio, followed by a $0.8 \times$. Libraries' size was analyzed using an Agilent Tapestation high sensitivity D5000 kit (Agilent Technologies) with an expected peak at $1000 \mathrm{bp}$ and absence of smaller primer peaks. Libraries were quantified using Qubit High-Sensitivity DNA kit and preparation kit, and libraries were constructed using Nextera XT DNA tagmentation (Illumina FC-131-1096) using $800 \mathrm{pg}$ of pooled cDNA library as input using index primers with format as done before (24). Amplified final libraries were purified twice with AMPure XP SPRI beads as before, with a volume ratio of $0.6 \times$ followed by $0.8 \times$ yielding library sizes with an average distribution of $650-750 \mathrm{bp}$. Libraries from 16 Seq-Well arrays were pooled and sequenced together using an Illumina NovaSeq 6000 S2 Reagent Kit v1.5 (100 cycles) using a paired-end read structure with custom read 1 primer: read 1: 20 bases with a 12-base cell barcode and 8-base UMI; read 2: 82 bases of transcript information, index 1 and index 2: 8 bases.

Single-cell RNA-Seq computational pipeline and analysis. Raw sequencing data were converted to demultiplexed FASTQ files using bcl2fastq2 based on Nextera N700 indices corresponding to individual arrays. Reads were then aligned to hg19 genome assembly and aligned using the Dropseq-tools pipeline on Terra (https:// app.terra.bio). Data were normalized and scaled using Seurat R package v.3.1.0 (https://satijalab.org/seurat/); 
A
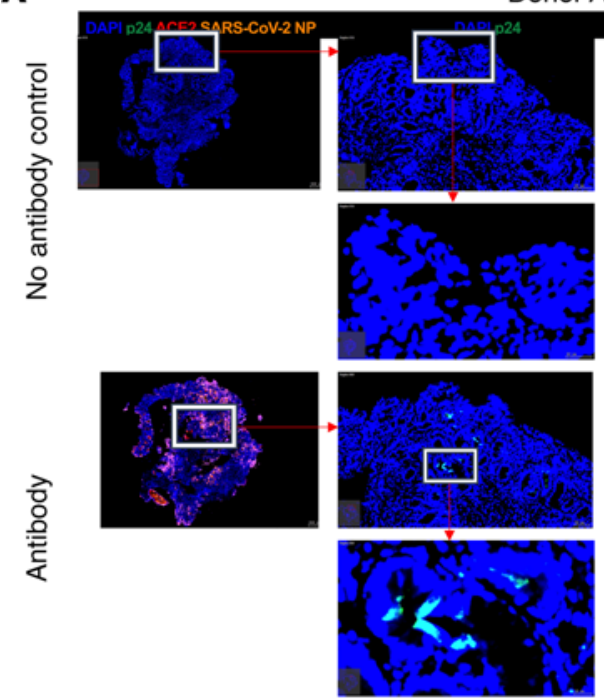

Donor A - $\left(\mathrm{HIV}^{+} \mathrm{ART}^{+}\right.$, SARS-CoV-2 $\left.{ }^{+}\right)$Duodenum
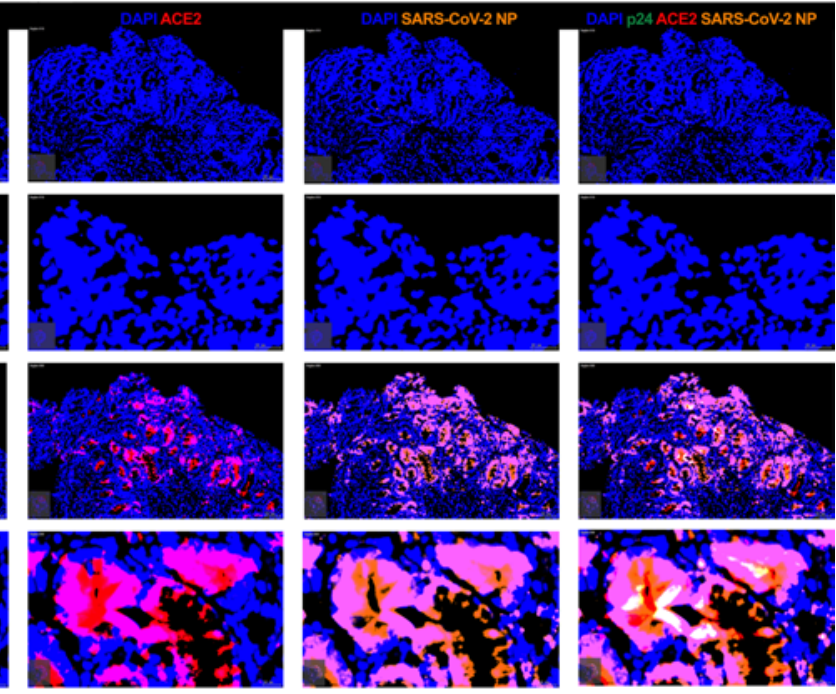

B

Donor B (pre-pandemic, HIV; SARS-CoV-2') Duodenum
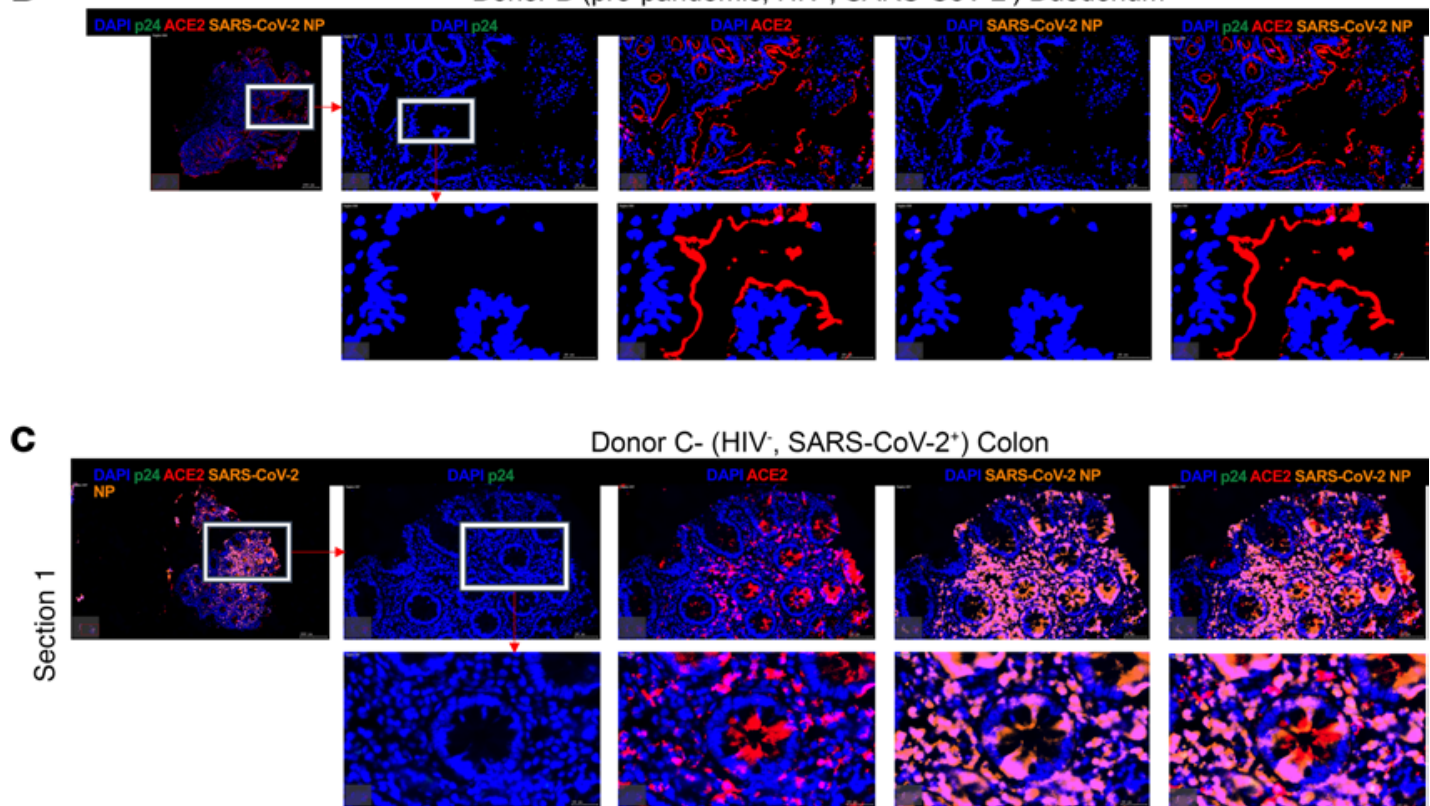

Donor C- (HIV, SARS-CoV-2+) Colon
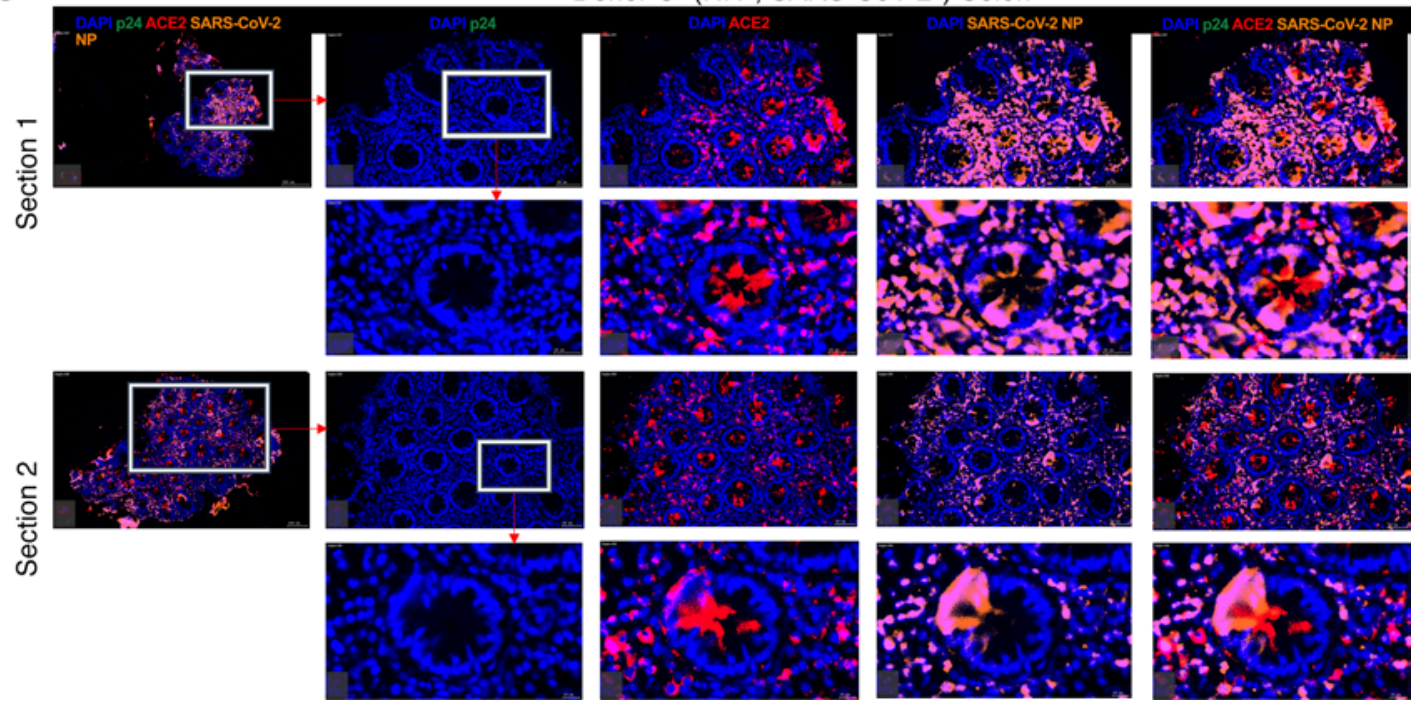

Figure 6. SARS-CoV-2 nucleocapsid detection overlaps with ACE2 expression in the small and large intestine. Representative fluorescence immunohistochemistry (F-IHC) images of duodenum and colon tissues showing HIV-p24 (green), ACE2 (red), SARS-CoV-2 nucleocapsid protein (orange), and DAPI (blue). (A) F-IHC image of a duodenum tissue from an HIV+SARS-CoV-2+ participants by PCR including no antibody control (top). (B) F-IHC image of a duodenum tissue from an HIV- SARS-CoV-2+ participant. (C) F-IHC image of a colon tissue from HIV-SARS-CoV-2+ with 2 sections shown from the same biopsy tissue. Scale bars are shown at the bottom right of each image. Scale bars: $20 \mu \mathrm{m}$ and $100 \mu \mathrm{m}$ for magnified inserts and main images, respectively. 
any cell with fewer than 750 UMIs or greater than 2500 UMIs was excluded from further analyses. This cellsby-genes matrix was then used to create a Seurat object. Cells with any gene expressed in fewer than 5 cells were discarded from downstream analysis, and any cell with at least 300 unique genes was retained. Cells with more than $20 \%$ of UMIs mapping to mitochondrial genes were then removed. These objects were then merged into 1 object for pre-processing and cell type identification. The combined Seurat object was log-normalized to UMI+1 using a scale factor of 10,000. We examined highly variable genes across all cells, yielding 2000 variable genes. Principal component analysis was applied to the cells to generate 100 principal components (PCs). Using the JackStraw function within Seurat, we identified significant PCs to be used for subsequent clustering and further dimensionality reduction. For $2 \mathrm{D}$ visualization and cell type clustering, we used a UMAP dimensionality reduction technique and with "min_dist" set to 0.5 and "n_neighbors" set to 30 . To identify clusters of transcriptionally similar cells, we employed unsupervised clustering as described above using the FindClusters tool within the Seurat R package with default parameters and k.param set to 10 and resolution set to 0.5 . We applied the default parameters with a shared nearest neighbor parameter optimized for each combined data set inside Monocle 3 package (V3.2.0) to construct single-cell pseudo-time trajectory to discover differential transitions. We used highly variable genes identified by Seurat to sort cells in pseudo-time order. The actual precursor determined the beginning of pseudo-time in the first round of "orderCells." UMAP was applied to reduce dimensional space, and the minimum spanning tree on cells was plotted by the visualization function "plot_cells" for Monocle 3. To further characterize substructure within cell types (for example, epithelial cells), we performed dimensionality reduction (PC analysis) and clustering over those cells alone. Differential expression analysis between the negative and positive groups of the same cell type was performed using the Seurat package FindAllMarkers in Seurat v3 (setting "test.use" to bimod). For each cluster, DEGs were generated relative to all the other cells. GO, gene set enrichment, and Kyoto Encyclopedia of Genes and Genomes pathway analyses from DEGs were performed using Metascape (https://metascape.org), which supports statistical analysis and visualization profiles for genes and gene clusters.

Histology and multicolor fluorescence immunohistochemistry. Formalin (4\%) fixed duodenum and colon tissue samples were embedded in paraffin, and a $4 \mu \mathrm{m}$ section of each was obtained on a glass slide. These sections were deparaffinized and incubated with anti-HIV p24 (clone: Kal-1, Dako), anti-SARS-CoV-2 nucleoprotein (clone: 40143-T-62, Sino Biological) anti-ACE2 (clone: ab15348; Abcam), anti-TMPRSS2 (clone: ab109131; Abcam), and anti-EpCAM (clone: ab71916; Abcam) followed by a secondary antibody incubation using the Opal 4-color manual IHC (PerkinElmer) as instructed by the manufacturer. For Opal fluorophores (PerkinElmer), FITC (product number FP1487001KT; PerkinElmer) was used for EpCAM and p24, Texas red (product number FP1488001KT; PerkinElmer) for ACE2, and Cy5 (product number FP1497001KT; PerkinElmer) for TMPRSS2 and SARS-CoV-2 nucleoprotein signal generation. DAPI was used as the nuclear counterstain. The sections were mounted with the Fluorescence Mounting Medium (catalog number S302380-2; Agilent Technologies) and cover-slipped, and the edges were sealed with nail polish. The slides were stored at $2^{\circ} \mathrm{C}-8^{\circ} \mathrm{C}$ until images were acquired.

Microscopy and quantitative image analysis. Images of the tissue sections were acquired using the TissueFAXS software (TissueGnostics) connected with a Zeiss Axio Observer Z1 inverted microscope (Olympus). The quantitative analysis of the cells of the different phenotypes within the images was done using the TissueQuest quantitation software (TissueGnostics)

Data availability. Next-generation sequencing data were deposited in National Center for Biotechnology Information's Gene Expression Omnibus under accession number GSE181877.

Statistics. Graphs were plotted using Prism 8.4.3 (GraphPad Inc.). Differences between groups were analyzed using the Seurat package FindAllMarkers in Seurat v3 (setting "test.use" to bimod). If any other specific test was used, it has been stated in the figure legends. A $P$ value less than 0.05 was considered significant.

Study approval. This study was approved by the Biomedical Research Ethics Committee of the University of KwaZulu-Natal (BE 021/13 and BE061/13). Participants gave written informed consent.

\section{Author contributions}

$\mathrm{RF}$ and OEA performed experiments and analyzed transcriptional data. SN supervised transcriptional analysis. NH, YZ, AS, AN, and IMM contributed to experimental work. NM consented participants and collected samples. DR and FK coordinated human tissue sample collection. WK, FGM, VTM, and FA contributed surgical human tissue samples. BB, THP, and AKS supervised data analysis. AKS, AL, and HNK provided intellectual input. RF, OEA, AL, and HNK prepared the manuscript. HNK conceptualized and supervised the work. 


\section{Acknowledgments}

HNK is supported by the Wellcome Trust $(202485 / \mathrm{Z} / 16 / \mathrm{Z})$ and the National Institute of Health (NIH) (R01DK126545). AL is supported by the Wellcome Trust (210662/Z/18/Z). This work was supported through the Sub-Saharan African Network for TB/HIV Research Excellence (SANTHE), a DELTAS Africa Initiative (grant DEL-15-006). The DELTAS Africa Initiative is an independent funding scheme of the African Academy of Sciences (AAS) Alliance for Accelerating Excellence in Science in Africa and supported by the New Partnership for Africa's Development Planning and Coordinating Agency (NEPAD Agency) with funding from the Wellcome Trust (grant 107752/Z/15/Z) and the United Kingdom government. HNK and AS were supported by SANTHE. AKS was supported, in part, by the Searle Scholars Program, the Beckman Young Investigator Program, the NIH (5U24AI118672, 2R01HL095791, 2U19AI089992, 1R01HL134539, 1R01AI138546), a Sloan Fellowship in Chemistry, and the Bill and Melinda Gates Foundation. The views expressed in this publication are those of the authors and not necessarily those of AAS, NEPAD Agency, Wellcome Trust, or the United Kingdom government.

Address correspondence to: Henrik N. Kløverpris, Africa Health Research Institute, K-RITH Tower Building, 719 Umbilo Road, Durban 4001, South Africa. Phone: 27.74.546.6625, 27.31.260.4186, or 45.29.720.910; Email: henrik.kloverpris@ahri.org.

1. Jin X, et al. Epidemiological, clinical and virological characteristics of 74 cases of coronavirus-infected disease 2019 (COVID-19) with gastrointestinal symptoms. Gut. 2020;69(6):1002-1009.

2. Lamers MM, et al. SARS-CoV-2 productively infects human gut enterocytes. Science. 2020;369(6499):50-54

3. Gaebler C, et al. Evolution of antibody immunity to SARS-CoV-2. Nature. 2021;591(7851):639-644.

4. Matheson NJ, Lehner PJ. How does SARS-CoV-2 cause COVID-19? Science. 2020;369(6503):510-511.

5. Xiao F, et al. Evidence for gastrointestinal infection of SARS-CoV-2. Gastroenterology. 2020;158(6):1831-1833.

6. Zang R, et al. TMPRSS2 and TMPRSS4 promote SARS-CoV-2 infection of human small intestinal enterocytes. Sci Immunol. 2020;5(47):eabc3582.

7. Liang W, et al. Diarrhoea may be underestimated: a missing link in 2019 novel coronavirus. Gut. 2020;69(6):1141-1143.

8. Hashimoto T, et al. ACE2 links amino acid malnutrition to microbial ecology and intestinal inflammation. Nature. 2012;487(7408):477-481.

9. Zhou J, et al. Infection of bat and human intestinal organoids by SARS-CoV-2. Nat Med. 2020;26(7):1077-1083

10. Mattapallil JJ, et al. Massive infection and loss of memory CD4+ T cells in multiple tissues during acute SIV infection. Nature. 2005;434(7037):1093-1097.

11. Veazey RS, Lackner AA. HIV swiftly guts the immune system. Nat Med. 2005;11(5):469-470.

12. Brenchley JM, et al. CD4+ T cell depletion during all stages of HIV disease occurs predominantly in the gastrointestinal tract. $J$ Exp Med. 2004;200(6):749-759.

13. Esterházy D, et al. Compartmentalized gut lymph node drainage dictates adaptive immune responses. Nature. 2019;569(7754):126-130.

14. Mann ER, et al. Compartment-specific immunity in the human gut: properties and functions of dendritic cells in the colon versus the ileum. Gut. 2016;65(2):256-270.

15. Trottein F, Sokol H. Potential causes and consequences of gastrointestinal disorders during a SARS-CoV-2 infection. Cell Rep. 2020;32(3):107915.

16. Boulle A, et al. Risk factors for COVID-2019 death in a population cohort study from the Western Cape Province, South Africa [published online August 29, 2020]. Clin Infect Dis. https://doi.org/10.1093/cid/ciaa1198.

17. Adepoju P. Tuberculosis and HIV responses threatened by COVID-19. Lancet HIV. 2020;3018(20):e319-e320.

18. Karim F, et al. HIV infection alters SARS-CoV-2 responsive immune parameters but not clinical outcomes in COVID-19 disease [preprint]. https://doi.org/10.1101/2020.11.23.20236828. Posted on medRxiv November 24, 2020.

19. Hoffmann C, et al. Immune deficiency is a risk factor for severe COVID-19 in people living with HIV. HIV Med. 2020;22(5):372-378

20. Ziegler CGK, et al. SARS-CoV-2 receptor ACE2 is an interferon-stimulated gene in human airway epithelial cells and is detected in specific cell subsets across tissues. Cell. 2020;181(5):1016-1035.

21. Onabajo OO, et al. Interferons and viruses induce a novel truncated ACE2 isoform and not the full-length SARS-CoV-2 receptor. Nat Genet. 2020;52(12):1283-1293.

22. Hoffmann M, et al. SARS-CoV-2 cell entry depends on ACE2 and TMPRSS2 and Is blocked by a clinically proven protease inhibitor. Cell. 2020;181(2):271-280.

23. Hughes TK, et al. Second-strand synthesis-based massively parallel scRNA-seq reveals cellular states and molecular features of human inflammatory skin pathologies. Immunity. 2020;53(4):878-894.

24. Gierahn TM, et al. Seq-Well: portable, low-cost RNA sequencing of single cells at high throughput. Nat Methods. 2017;14(4):395-398.

25. Muus C, et al. Single-cell meta-analysis of SARS-CoV-2 entry genes across tissues and demographics. Nat Med. 2021;27(3):546-559.

26. Letko M, et al. Functional assessment of cell entry and receptor usage for SARS-CoV-2 and other lineage B betacoronaviruses. Nat Microbiol. 2020;5(4):562-569.

27. Wu N, et al. The interferon stimulated gene 20 protein (ISG20) is an innate defense antiviral factor that discriminates self versus non-self translation. PLoS Pathog. 2019;15(10):e1008093.

28. Wurster AL, et al. The biology of Stat4 and Stat6. Oncogene. 2000;19(21):2577-2584. 
29. Almasan A, Ashkenazi A. Apo2L/TRAIL: apoptosis signaling, biology, and potential for cancer therapy. Cytokine Growth Factor Rev. 2003;14(3-4):337-348.

30. Cantarella G, et al. Neutralization of TNFSF10 ameliorates functional outcome in a murine model of Alzheimer's disease. Brain. 2015;138(1):203-216.

31. Khawar MB, et al. IL-32: A novel pluripotent inflammatory interleukin, towards gastric inflammation, gastric cancer, and chronic rhino sinusitis. Mediators Inflamm. 2016;2016:8413768.

32. Brenchley JM, Douek DC. HIV infection and the gastrointestinal immune system. Mucosal Immunol. 2008;1(1):23-30.

33. Lee JJ, et al. Relative abundance of SARS-CoV-2 entry genes in the enterocytes of the lower gastrointestinal tract. Genes (Basel). 2020;11(6):645.

34. Liao M, et al. Single-cell landscape of bronchoalveolar immune cells in patients with COVID-19. Nat Med. 2020;26(6):842-844.

35. Vuille-Dit-Bille RN, et al. Human intestine luminal ACE2 and amino acid transporter expression increased by ACE-inhibitors. Amino Acids. 2015;47(4):693-705.

36. Camargo SMR, et al. Tissue-specific amino acid transporter partners ACE2 and collectrin differentially interact with hartnup mutations. Gastroenterology. 2009;136(3):872-882.

37. Monaco CL, et al. Altered virome and bacterial microbiome in human immunodeficiency virus-associated acquired immunodeficiency syndrome HHS public access. Cell Host Microbe. 2016;9(193):311-322.

38. Santisteban MM, et al. Brain-gut-bone marrow axis: implications for hypertension and related therapeutics. Circ Res. 2016;118(8):1327-1336.

39. Kim S, et al. Altered gut microbiome profile in patients with pulmonary arterial hypertension. Hypertension. 2020;75(4):1063-1071.

40. Clark SA, et al. SARS-CoV-2 evolution in an immunocompromised host reveals shared neutralization escape mechanisms. Cell. 2021;184(10):2605-2617.

41. Kemp SA, et al. SARS-CoV-2 evolution during treatment of chronic infection. Nature. 2021;592(7853):277-282.

42. Sigel K, et al. Coronavirus 2019 and people living with human immunodeficiency virus: outcomes for hospitalized patients in New York City. Clin Infect Dis. 2020;71(11):2933-2938.

43. Vizcarra P, et al. Description of COVID-19 in HIV-infected individuals: a single-centre, prospective cohort. Lancet HIV. 2020;7(8):e554-e564.

44. San JE, et al. Transmission dynamics of SARS-CoV-2 within-host diversity in two major hospital outbreaks in South Africa. Virus Evol. 2021;7(1):veab041.

45. Tegally $\mathrm{H}$, et al. Emergence of a SARS-CoV-2 variant of concern with mutations in spike glycoprotein. Nature. $2021 ; 592(7854): 438$ 